\title{
The Lorentz Process Converges to a Random Flight Process
}

\author{
Herbert Spohn* \\ Joseph Henry Laboratories, Princeton University, Princeton, New Jersey 08540, USA
}

\begin{abstract}
The Lorentz process is the stochastic process defined by a particle moving, according to Newton's law of motion, through static scatterers distributed according to some probability measure in space. We consider the Boltzmann-Grad limit: The density of scatterers increases to infinity and at the same time the diameter of the scatterers decreases to zero in such a way that the mean free path of the particle is kept constant. We show that the Lorentz process converges in the weak*-topology of regular Borel measures on the paths space to some stochastic process. The limit process is Markovian if and only if the rescaled density of scatterers converges in probability to its mean. In that case the limit process is a (spatially inhomogeneous) random flight process.
\end{abstract}

\section{Introduction}

H. A. Lorentz [1] considered the following simple mechanical model for the motion of an electron through a solid: A single particle moves according to the laws of Newtonian mechanics through fixed scatterers ("the solid") located in a random fashion throughout space. We call the associated stochastic process - to be defined more precisely in a moment - the Lorentz process.

Let $x=(q, p) \in \mathbb{R}^{3} \times \mathbb{R}^{3}$ denote the position and momentum of the moving particle. We assume that it is enclosed in a bounded region $\Lambda \subset \mathbb{R}^{3}$ with smooth boundaries $\partial \Lambda$. Upon colliding with the boundary the particle becomes specularly reflected. The particle interacts with the scatterers by a spherically symmetric potential $V_{R}$ of finite range $R$. The potential may have a hard core of radius $a$, $0 \leqq a \leqq R$, and is twice continuously differentiable for $a \leqq|q|$. Let $(q)_{j}=\left(q_{1}, \ldots q_{j}\right) \in$ $\Lambda^{j}$ be a configuration of $j$ scatterers. Then Hamilton's equation of motion, subject to the boundary conditions coming from the hard core and the boundary of $\Lambda$,

$$
\frac{d}{d t} q(t)=p(t)
$$

* On leave of absence of Fachbereich Physik der Universität München. Work supported by a DFG fellowship 


$$
\frac{d}{d t} p(t)=-\sum_{i=1}^{j} \frac{\partial}{\partial q} V_{R}\left(q(t)-q_{i}\right)
$$

have unique global solutions $T_{t}^{(q)}{ }_{j} x$ for all initial values $x \in \Lambda \times \mathbb{R}^{3}$ such that $\left|q-q_{i}\right| \geqq a, i=1, \ldots, j$. For notational simplicity we extend $T_{t}^{(q)_{j}}$ to all $\Lambda \times \mathbb{R}^{3}$ by $T_{t}^{(q) j} x=x$ whenever $\left|q-q_{i}\right|<a$ for some $i$. Then $T_{t}^{(q)_{j}}$ defines a one-parameter group of canonical transformations. In particular, $T_{t}^{(q)_{j}}$ preserves the Lebesque measure on $\Lambda \times \mathbb{R}^{3}$.

Inside $\Lambda$ the scatterers are randomly distributed according to some probability measure $\mu^{(R)}$ on $\Gamma=\bigcup_{j \geq 0} \Lambda^{j}$. Since scatterers are supposed to be identical, $\mu^{(R)} \uparrow \Lambda_{j}$ is symmetric. (Equivalently, we could think of $\mu^{(R)}$ as a measure on all finite subsets of $\Lambda$.) Then, with $(q)_{j} \in \Gamma$

$$
x\left(t, x_{0}\right):(q)_{j} \mapsto T_{t}^{(q)} x_{0}
$$

is called the Lorentz process with respect to $\mu^{(R)}$ starting at $x_{0}$ and taking values in $\Lambda \times \mathbb{R}^{3}$.

(Here a remark on generality is in order: Clearly, we did not define the most general Lorentz process. One could think of other boundary conditions. The potential could be chosen in a more general way. We are not restricted to three dimensional space. The particle could move in an external force field, etc. We will comment on some of the more interesting extensions in the final section. We hope that the chosen assumptions illustrate the method best.)

Having defined the Lorentz process, one is optimistic enough to ask some simple questions. Of particular interest to physicists are quantities related to the transport properties of the Lorentz model. E.g., one would like to investigate the mean square distance travelled by the particle in time $t$ starting at $q_{0}$ and having uniform momentum distribution on $\left\{\left|p_{0}\right|=v\right\}$. With no boundaries and a uniform low density of scatterers, on physical grounds, one expects the same long time behaviour as in the diffusion (Brownian) process, i.e. proportional to $t$. This conjecture is backed up by machine calculations [2]. However, even for the simplest distribution of scatterers, namely according to the equilibrium (Gibbs) measure of a noninteracting gas, no such result has been proved. The Lorentz process seems simply to be too complicated.

In view of such a lamentable situation, one could hope to approximate the Lorentz process by some process which is easier to handle. (This is precisely what Lorentz did along the lines of Boltzmann, although of course not with the present day technical refinement.) Intuitively, the directions of the particle become randomized through collision with a scatterer. Of course, the particle might collide with the same scatterer twice which produces a memory effect. But, if the range $R$ of the potential is small, the probability of such recollisions should be small also. Therefore, one expects the Lorentz process for small $R$ to be close to a random flight process where a particle leaves $q_{0}$ with momentum $p_{0}$, flies in a time $t$ a path of length $\left|p_{0}\right| t$, suffers shocks with a certain probability and is at each shock deviated randomly with a distribution depending on the differential cross section of the potential. 
The task of the present paper is to make these ideas precise. We let the range $R$ of the potential $V_{R}$ decrease to zero. This would result in the free motion for the particle. We therefore choose a sequence of probability measures $\mu^{(R)}$ on $\Gamma$ in such a way that at the same time as $R \rightarrow 0$ the density of the scatterers increases to infinity such that the mean free path of the particle remains constant. (This is the Boltzmann-Grad limit.) Under suitable conditions on the sequence $\mu^{(R)}$, we show then that the Lorentz process converges to some stochastic process. More precisely, the sequence of regular Borel probability measures $P^{g(R)}$ on the space of paths $\Omega=\underset{t \geqq 0}{X}\left(\Lambda \times \dot{\mathbb{R}}^{3}\right)_{t}\left(\dot{\mathbb{R}}^{3}\right.$ is the one-point compactification of $\left.\mathbb{R}^{3}\right)$ induced by the Lorentz process with respect to $\mu^{(R)}$ with absolutely continuous starting probability $g d x$ converges in the weak*-topology of regular Borel measures on $\Omega$ to a probability measure $P^{g}$. The stochastic process associated with $P^{g}$ is Markovian if and only if the rescaled density of scatterers in every open subset of $\Lambda$ converges in probability to its mean. In that case the Markov process $P^{g}$ is a (spatially inhomogeneous) random flight process.

Gallavotti $[3,4]$ proved in the special case of Poisson distributed hard sphere scatterers in two dimensions that the single time distribution converges to the single time distribution of the random flight process $P^{g}$. We employ here a method which was developed by Lanford [5] to derive the Boltzmann equation for a hard sphere gas (for short times). We also rely on the extension by King [6] to smooth potentials. It turns out that Lanford's method is a very powerful tool to prove the convergence of a large class of processes in the Boltzmann-Grad limit. We will comment on related work in the last section.

\section{Correlation Functions and the BBGKY Hierarchy}

We want to study the convergence of the Lorentz process $x\left(t, x_{0}\right)$ for a certain sequence of measures $\mu^{(R)}$ on $\Gamma$ as $R \rightarrow 0$. The crucial point in order to control the limit is to think of the Lorentz process as a mechanical system (i.e. a system of many particles with a rather trivial time evolution) in the first place. Therefore we investigate the time evolution of correlation functions and their limit as $R \rightarrow 0$. Later on we will have the task to translate the "mechanical" information back to information about the process itself.

The first part of this section is well known from statistical mechanics $[7,8]$. Here, however, we have to adapt the various notions to the Lorentz process and at the same time we want to introduce some notation.

Let $Y_{j}$ be the Banach space $L^{1}\left(\Lambda \times \mathbb{R}^{3}\right) \otimes C\left(\Lambda^{j}\right)_{\text {symm }}$ with norm

$$
\left\|\rho_{j}\right\|_{j}=\sup _{(q)_{j} \in \Lambda^{j}} \int_{\Lambda \times \mathbb{R}^{3}} d x\left|\rho_{j}\left(x,(q)_{j}\right)\right|
$$

$j=0,1, \ldots \rho_{j}$ is symmetric in $\left(q_{1}, \ldots, q_{j}\right)$. Let $\rho=\left(\rho_{0}, \rho_{1}, \ldots\right)$ with $\rho_{j} \in Y_{j}$ and let $Y_{z}$ be the Banach space of such sequences with norm

$$
\|\rho\|_{z}=\sup _{j} z^{-j}\left\|\rho_{j}\right\|_{j}
$$

$z>0$. 
We assume that the joint probability distribution for the moving particle and the scatterers is given by $f \in Y_{z}$ which is positive, symmetric in $\left(q_{1}, \ldots, q_{j}\right)$ and normalized to

$$
\sum_{j=0}^{\infty} \int d x d(q)_{j} \frac{1}{j !} f_{j}\left(x,(q)_{j}\right)=1
$$

Then the correlation functions for $f$ are defined by

$$
\rho_{j}\left(x,(q)_{j}\right)=\sum_{m=0}^{\infty} \int_{A^{m}} \frac{1}{m !} d\left(q^{\prime}\right)_{m} f_{j+m}\left(x,(q)_{j},\left(q^{\prime}\right)_{m}\right) .
$$

Clearly $\rho \in Y_{Z}$. We extend this definition by linearity to all of $Y_{Z}$. The relation inverse to (2.3) is given by

$$
f_{j}\left(x,(q)_{j}\right)=\sum_{m=0}^{\infty}(-1)^{m} \int_{A^{m}} \frac{1}{m !} d\left(q^{\prime}\right)_{m} \rho_{j+m}\left(x,(q)_{j},\left(q^{\prime}\right)_{m}\right) .
$$

The flows $T_{t}^{(q)_{j}}$ induce a time evolution on $Y_{z}$ by $f_{j}\left(x,(q)_{j}\right) \mapsto f_{j}\left(T_{-t}^{(q)_{j}} x,(q)_{j}\right)$ which in term defines an evolution $\rho \mapsto V_{t}^{(R)} \rho$ on $Y_{z} \cdot V_{t}^{(R)}$ is a strongly continuous bounded group. (For correlation functions $\rho$ coming from a positive $f V_{t}^{(R)}$ is isometric.)

The limit we want to consider is more conveniently studied for the reduced correlation functions. For $f \in Y_{z}$ they are defined by

$$
\left(Q^{(R)} \rho\right)_{j}\left(x,(q)_{j}\right)=\sum_{m=0}^{\infty} \int_{\left\{\left|q-q_{1}^{\prime}\right| \geqq R, \ldots,\left|q-q_{m}^{\prime}\right| \geqq R\right\}} \frac{1}{m !} d\left(q^{\prime}\right)_{m} f_{j+m}\left(x,(q)_{j},\left(q^{\prime}\right)_{m}\right)
$$

$R \geqq 0$. By a straight forward computation

$$
\rho_{j}\left(x,(q)_{j}\right)=\sum_{m=0}^{\infty} \int_{\left\{\left|q-q^{\prime}\right| \leqq R, \ldots,\left|q-q_{m}^{\prime}\right| \leqq R\right\}} \frac{1}{m !} d\left(q^{\prime}\right)_{m}\left(Q^{(R)} \rho\right)_{j+m}\left(x,(q)_{j},\left(q^{\prime}\right)_{m}\right)
$$

with inverse relation

$$
\left(Q^{(R)} \rho\right)_{j}\left(x,(q)_{j}\right)=\sum_{m=0}^{\infty}(-1)^{m} \int_{\left\{\left|q-q^{\prime}\right| \leqq R, \ldots,\left|q-q_{m}^{\prime}\right| \leqq R\right\}} \frac{1}{m !} d\left(q^{\prime}\right)_{m} \rho_{j+m}\left(x,(q)_{j},\left(q^{\prime}\right)_{m}\right) .
$$

Therefore $Q^{(R)}: Y_{z} \rightarrow Y_{z}$ is bounded and invertible.

We have

$$
\left\|Q^{(R)}-1\right\|_{z} \leqq \exp \left[\frac{4 \pi}{3} R^{3} z\right]-1 .
$$

The reduced correlation functions evolve as $Q^{(R)} \rho \mapsto Q^{(R)} V_{t}^{(R)} \rho=U_{t}^{(R)} Q^{(R)} \rho$. $U_{t}^{(R)}$ is a strongly continuous bounded group.

To determine the generator of $U_{t}^{(R)}$ we assume that $f$ is continuously differentiable and of compact support. Then

$$
\begin{aligned}
& \left.\left(\frac{d}{d t} U_{t}^{(R)}\right)_{j}\right|_{t=0}\left(x,(q)_{j}\right)=\sum_{m=0}^{\infty} \int_{\left\{\left|q-q^{\prime}\right| \geqq R, \ldots,\left|q-q_{m}^{\prime}\right| \geqq R\right\}} \frac{1}{m !} d\left(q^{\prime}\right)_{m} \\
& \left(p \frac{\partial}{\partial q}+\sum_{i=1}^{j+m} \frac{\partial}{\partial q} V\left(q-q_{i}\right) \frac{\partial}{\partial p}\right) f_{j+m}\left(x,(q)_{j},\left(q^{\prime}\right)_{m}\right) .
\end{aligned}
$$


Using

$$
\begin{aligned}
\int_{\left|q-q_{m}\right| \geqq R} d q_{m} p \frac{\partial}{\partial q} f\left(q, q_{m}\right)= & p \frac{\partial}{\partial q_{\left|q-q_{m}\right| \geqq R}} \int_{S^{2}} d q_{m} f\left(q, q_{m}\right) \\
& +R^{2} \int_{S^{2}} d \sigma(\hat{\omega}) \hat{\omega} \cdot p f(q, q+R \hat{\omega}),
\end{aligned}
$$

where $\hat{\omega}$ is a unit vector of the 2-sphere $S^{2}$ and $d \hat{\omega}$ is the surface measure, and the symmetry of $f_{j+m}$, we obtain

$$
\begin{aligned}
&\left.\left(\frac{d}{d t} U_{t}^{(R)} \rho\right)\right|_{j}\left(x,(q)_{j}\right)=\left.\frac{\partial}{\partial t} \rho_{j}\left(T_{-t}^{(q)} x,(q)_{j}\right)\right|_{t=0} \\
&-R^{2} \int_{S^{2} \cap\{q+R \hat{\omega} \in \Lambda\}} d \sigma(\hat{\omega}) \hat{\omega} \cdot p \rho_{j+1}\left(x,(q)_{j}, q+R \hat{\omega}\right) \\
& \cdot \delta^{(a)}\left(x,(q)_{j}\right)
\end{aligned}
$$

with $\delta^{(a)}\left(x,(q)_{j}\right)=0$ whenever $\left|q-q_{i}\right|<a, i=1, \ldots, j$, and $\delta^{(a)}\left(x,(q)_{j}\right)=1$ otherwise. The set of equations (2.10) is called the BBGKY-hierarchy. It should be noticed that the boundary conditions (hard core and the boundary of $\Lambda$ ) are contained in the definition of $T_{t}^{(q)}$.

We define on $Y_{z}$ the one parameter group of isometries

$$
\left(S^{(R)}(t) \rho\right)_{j}=\rho_{j}\left(T_{-t}^{(q)_{j}} x,(q)_{j}\right)
$$

and for $\rho \in Y_{z}$ with bounded momentum support

$$
\begin{aligned}
& \left(C^{(R)} \rho\right)_{j}=C_{j, j+1}^{(R)} \rho_{j+1} \\
& \left(C_{j, j+1}^{(R)} \rho_{j+1}\right)\left(x,(q)_{j}\right)=-\int_{S^{2} \cap\{q+R \hat{\omega} \in \Lambda\}} d \sigma(\hat{\omega}) \hat{\omega} \cdot p \rho_{j+1}\left(x,(q)_{j}, q+R \hat{\omega}\right) \\
& \cdot(a)\left(x,(q)_{j}\right) .
\end{aligned}
$$

In (2.10) we would like to consider the collision term $C^{(R)}$ as a perturbation. However, on $Y_{z} C^{(R)}$ is unbounded. We therefore construct an $U_{t}^{(R)}$-invariant subspace of $Y_{z}$ on which $C^{(R)}$ becomes bounded. Let $Y_{z}^{E}$ be the set of all functions $\rho \in Y_{z}$ such that $\rho_{j}$ vanishes on the complement of $\left\{x \in \Lambda \times \mathbb{R}^{3},(q)_{j} \in \Lambda^{j}|| q_{i}\right.$ $q_{i^{\prime}}\left|\geqq R, i \neq i^{\prime}=1, \ldots, j,\right| q \mid<a$, and for $\left.|q| \geqq a \frac{p^{2}}{2}+\sum_{i=1}^{j} V_{R}\left(q-q_{i}\right) \leqq E\right\}, E>0, j=$ $0,1, \ldots$ Then $Y_{z}^{E}$ is a closed $U_{t}^{(R)}$-invariant subspace. For $\rho \in Y_{z}^{E}$

$$
\left\|C^{(R)} \rho\right\|_{z} \leqq\|\rho\|_{z} z\left(2\left(E+\sup _{a \leqq|q|}|V(|q|)|\right)\right)^{1 / 2} .
$$

This proves

Theorem 1. Let $\rho \in Y_{z}^{E}$. Then for all $t \geqq 0$

$$
\begin{aligned}
U_{t}^{(R)} \rho=\sum_{k=0}^{\infty} R^{2 k} \int_{0 \leqq t_{k} \ldots \leqq t_{1} \leqq t} d t_{1} \ldots d t_{k} S^{(R)}\left(t-t_{1}\right) C^{(R)} \\
\cdot S^{(R)}\left(t_{2}-t_{1}\right) \ldots C^{(R)} S^{(R)}\left(t_{k}\right) \rho .
\end{aligned}
$$

The series (2.14) converges in norm. With reversed time integration (2.14) holds also for $t \leqq 0$. 


\section{The Boltzmann-Grad Limit}

The Boltzmann Grad limit is the following limiting procedure: In the BBGKYhierarchy (2.10) we consider a sequence of correlation functions $\rho^{(R)}, R \rightarrow 0$, such that the rescaled correlation functions $R^{2 j} \rho_{j}^{(R)}=r_{j}^{(R)}$ have limits $r_{j}$. Then, in particular, $\rho_{1}\left(x, q_{1}\right) \sim R^{-2} r_{1}\left(x, q_{1}\right)$ which means that the density of scatterers goes to infinity. (However, the volume $R^{3} \rho_{1}$ occupied by scatterers goes to zero.) We scale the potential as $V_{R}(q)=V\left(\frac{q}{R}\right)$, where $V$ is a fixed potential of range 1 . Under the scaling the mapping from incoming to outgoing momentum is preserved. If the moving particle hits a scatterer it might happen that the collision time is infinite, i.e. the particle needs an infinite time to reach the point closest to the center of the scatterer. Twice differentiability does not exclude, for fixed incoming momentum $p$, a dense set of infinite collision times, which would cause some difficulties for proving the Boltzmann-Grad limit. To exclude this to happen we assume that

$\left(^{*}\right) E-V(r)-\frac{J^{2}}{2 r^{2}}=0, \frac{J^{2}}{r^{3}}-\frac{d}{d r} V(r)=0$,

$r=|q|, a \leqq r \leqq 1$, is fulfilled simultaneously only for a finite number of values $E$ and $J$.

We first describe what the Boltzmann-Grad limit will look like and give then a theorem stating how the limit is approached. For the convergence of the Lorentz process it is of importance to ensure the "propagation of initial conditions" (assumed convergence at $t=0$ implies the same kind of convergence at later time).

Let $\tilde{Y}_{j}^{E}=C\left(\Lambda \times\left\{\frac{p^{2}}{2} \leqq E\right\}\right) \times C\left(\Lambda^{j}\right)_{\text {symm }}$ considered as a subset of $Y_{j}$ and let $\tilde{Y}_{z}^{E}$ be the Banach space of sequences $r=\left(r_{0}, r_{1}, \ldots\right)$ such that $r_{j} \in \tilde{Y}_{j}^{E}$ and

$\sup \sup z^{-j}\left|r_{j}\left(x,(q)_{j}\right)\right|=\|r\|_{z}<\infty$.

$j \quad x,(q)_{j}$

Let $S(t)$ be the one-parameter group of isometries on $\tilde{Y}_{z}^{E}$ defined by

$$
(S(t) r)_{j}\left(x,(q)_{j}\right)=r_{j}\left(T_{-t}^{(0)} x,(q)_{j}\right)
$$

with generator $L$,

$$
(\operatorname{Lr})_{j}\left(x,(q)_{j}\right)=-p \frac{\partial}{\partial q} r\left(x,(q)_{j}\right)
$$

for $r \in D(L)$. ((0) stands for no scatterer, i.e. $T_{t}^{(0)}$ is the free motion with specular reflection at the boundary.)

Let

$$
(C r)_{j}=C_{j, j+1} r_{j+1}
$$

and

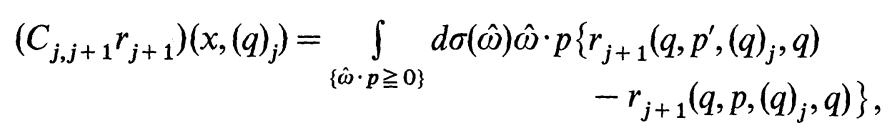


where $\hat{\omega}$ points inwards for $q \in \partial \Lambda$, and where $p^{\prime}$ is the outgoing momentum to the incoming momentum $p$ colliding with the potential $V$ at the point $\hat{\omega}$. $C$ generates a strongly continuous, bounded semigroup on $\tilde{Y}_{z}^{E}$, therefore, so does $L+C$ on $D(L)$ denoted by $U_{t}=e^{(L+C) t}, t \geqq 0$. For the past we define the strongly continuous bounded semigroup $U_{t}=e^{(L-C) t}, t \leqq 0$.

Let $\Gamma_{j}(t)=\left\{\left(x,(q)_{j}\right) \in \Lambda \times \mathbb{R}^{3} \times \Lambda^{j} \mid q \neq q_{i} \neq q_{i^{\prime}}, i \neq i^{\prime}=1, \ldots, j\right.$ and the curve $s \mapsto$ position of $T_{-s}^{(0)} x, 0 \leqq s \leqq t$ for $t \geqq 0, t \leqq s \leqq 0$ for $t \leqq 0$, does not intersect $q_{1}, \ldots, q_{j}$ and does not intersect itself $\}$. If $\left(x,(q)_{j}\right) \in \Gamma_{j}(t)$ then we can choose $R$ small enough such that $T_{-s}^{(q)}$ suffers no collision within $0 \leqq s \leqq t($ resp. $t \leqq s \leqq 0)$. We emphasize that $\Gamma_{j}(t)$ is not symmetric under time-reversal, i.e. $\Gamma_{j}(t) \neq \Gamma_{j}(-t)$, $t \neq 0$.

Theorem 2. Let $V$ satisfy $\left({ }^{*}\right)$ and let $\rho^{(R)} \in Y_{z / R^{2}}^{E}$ be a sequence of correlation functions such that

(1) $\left\|\rho^{(R)}\right\|_{z / R^{2}}$ is uniformly bounded in $R$

(2) $R^{2 j} \rho_{j}^{(R)}$ converges to $r_{j}$ with $r \in \tilde{Y}_{z}^{E}$ uniformly on compact sets of $\Gamma_{j}\left(t_{0}-t\right)$ as $R \rightarrow 0,|t| \leqq\left|t_{0}\right|$.

Then $R^{2 j}\left(U_{t}^{(R)} \rho^{(R)}\right)_{j}$ converges to $\left(U_{t} r\right)_{j}$ uniformly on compact sets of $\Gamma_{j}\left(t_{0}\right)$ as $R \rightarrow 0$.

The proof proceeds in three steps. The main idea is to analyze a single scattering event and to show that in the limit the probability for recollisions goes to zero.

Lemma 1. Let $\Sigma\left(x, q_{1}, R\right) \subset S^{2}$ be the set of all $\hat{\omega}$ 's such that if a scaterer is adjoined at $q_{2}=q+R \hat{\omega}$ the particle, moving according to $T_{-s}^{\left(q_{1}, q_{2}\right)} x$, will collide within time $-t, t \geqq 0$, with either the scatterer at $q_{1}$ or the scatterer at $q_{2}$ after having hit the boundary of $\Lambda$ at least once. Let $K_{1} \subset \Gamma_{1}(t)$ be compact. Then

$$
\lim _{\boldsymbol{R} \rightarrow 0} \sup _{\left(x, q_{1}\right) \in \mathbf{K}_{1}} \int_{\Sigma\left(x, q_{1}, \boldsymbol{R}\right)} d \sigma(\hat{\omega})=0 .
$$

Proof. Since $K_{1}$ is compact, we can choose $R$ small enough such that scatterer $q_{1}$ and scatterer $q_{2}=q+R \hat{\omega}$ do not overlap for all $\left(x, q_{1}\right) \in K_{1}$. If $\hat{\omega} \cdot p \geqq 0$, the particle and scatterer $q_{2}$ do not collide immediately. For $R$ small enough $\Sigma\left(x, q_{1}\right.$, $R) \cap\{\hat{\omega} \mid \hat{\omega} \cdot p \geqq 0\}=\varnothing$. Let $\hat{\omega} \cdot p \leqq 0$. Then the particle will immediately collide with scatterer $q_{2}$ backwards in time. If the collision is completed within time $-t$, let $p^{\prime}=F_{p}(\hat{\omega})$ be the momentum after collision. Then we can find a finite number of cones, the union of which is denoted by $\mathscr{C}(c R)$, each of them having vertex at the origin, axis along a line of free motion hitting $q_{1}$ or rehitting $q_{2}$ within $-t$ and opening $c R$ such that $\Sigma\left(x, q_{1}, R\right) \subset F_{p}^{-1}(\mathscr{C}(c R))$. By dominated convergence

$$
\lim _{R \rightarrow 0} \int_{\Sigma\left(x, q_{1}, R\right)} d \sigma(\hat{\omega})=\int_{F_{p}^{-1}(\mathscr{L}(0))} d \sigma(\hat{\omega}) .
$$

$\mathscr{C}(0)$ is the set of vectors pointing along the lines of free motion leaving $q$ and hitting $q_{1}$ or rehitting $q$ within time $-t$. By conservation of energy $|p|=\left|p^{\prime}\right|$. By conservation of angular momentum $p, p^{\prime}$ and $\hat{\omega}$ lie in a common plane. Fix the angle between $\hat{\omega}$ and $p$ and rotate $\hat{\omega}$ around $p$. Then $p^{\prime}$ rotates along a cone. If $p$ is parallel to $p^{\prime}$ there is no further collision up to $-t$. If $p$ is not parallel to $p^{\prime}$, then there are only finitely many $\hat{\omega}$ 's such that $p^{\prime} \in \mathscr{C}(0)$. Therefore, by Fubini's 
theorem,

$$
\int_{F_{\bar{p}} 1(\mathscr{C}(0))} d \sigma(\hat{\omega})=0
$$

The collision time scales proportional to $R$. By assumption $\left({ }^{*}\right)$ on the potential for given incident $p$ there are finitely many $\hat{\omega}$ 's for which there is an infinite collision time. We can choose an open set $\Sigma_{\infty}(p) \subset S^{2}$ of measure smaller than $\varepsilon$ such that the collision time is uniformly bounded on $S^{2} \backslash \Sigma_{\infty}(p)$ for all $\left(x, q_{1}\right) \in K_{1}$. The map $\left(x, q_{1}\right) \mapsto \int_{\Sigma\left(x, q_{1}, R\right) \backslash \Sigma_{\infty}(p)} d \sigma(\hat{\omega})$ is continuous on $K_{1}$ and, for $R$ small enough such that the collision times are smaller than $t$, monotonely decreasing. Therefore by Dini's theorem we have uniform convergence on $K_{1}$.

Lemma 2. Assume (1) and (2). Then for $t_{1}, t_{2}>0, t_{1}+t_{2} \leqq t,\left(S^{(R)}\left(t_{1}\right) C^{(R)} S^{(R)}\right.$ $\left.\left(t_{2}\right) r^{(R)}\right)_{j}$ converges to $\left(S\left(t_{1}\right) C S\left(t_{2}\right) r\right)_{j}$ uniformly on compact sets of $\Gamma_{j}\left(t_{0}\right)$ as $R \rightarrow 0$.

Proof. We use the following notation: $\left(q^{\prime}, p^{\prime}\right)=T_{-t_{1}}^{(q)_{j}}(q, p)$ and $\left(q^{\prime \prime}, p^{\prime \prime}\right)=T_{-t_{2}}^{(q)_{j+1}}\left(q^{\prime}, p^{\prime}\right)$ with scatterer adjoined at $q_{j+1}=q^{\prime}+R \hat{\omega}$. Then

$$
\left(S^{(R)}\left(t_{1}\right) C^{(R)} S^{(R)}\left(t_{2}\right) r^{(R)}\right)_{j}\left(x,(q)_{j}\right)=-\int_{S^{2}} d \sigma(\hat{\omega}) \hat{\omega} \cdot p r_{j+1}^{(R)}\left(q^{\prime \prime}, p^{\prime \prime},(q)_{j}, q^{\prime}+R \hat{\omega}\right) .
$$

Let $K_{j} \subset \Gamma_{j}\left(t_{0}\right)$ be compact. Then we can choose $R$ small enough such that the particle will not collide with any of the scatterers at $q_{1}, \ldots, q_{j}$ within time $-t_{1}$ for all $\left(x,(q)_{j}\right) \in K_{j}$. Furthermore, by induction to Lemma 1, there exists for small enough $R$ a compact set $G\left(x,(q)_{j}, R\right) \subset S^{2}$ such that for all $\hat{\omega} \in G\left(x,(q)_{j}, R\right)$ and all $\left(x,(q)_{j}\right) \in K_{j}$ the collision time with $q_{j+1}=q^{\prime}+R \hat{\omega}$ is smaller than $t_{2}$, (b) for all $\hat{\omega} \in G\left(x,(q)_{j}, R\right)$ and all $\left(x,(q)_{j}\right) \in K_{j}$ there is besides the possible initial collision between the particle and scatterer $q_{j+1}$ neither a collision between the particle and scatterers $q_{1}, \ldots, q_{j}$ nor a collision between the particle and the scatterer $q_{j+1}$ after hitting at least once the boundary of $\Lambda$ within time $-t_{2},(c)$

$$
\int_{S^{2} \backslash G\left(x,(q)_{j}, R\right)} d \sigma(\hat{\omega})<\varepsilon
$$

for all $\left(x,(q)_{j}\right) \in K_{j}$. By the uniform bound (1) it therefore suffices to study

$$
-\int_{G(x,(q) j, R)} d \sigma(\hat{\omega}) \hat{\omega} \cdot p r_{j+1}^{(R)}\left(q^{\prime \prime}, p^{\prime \prime},(q)_{j}, q^{\prime}+R \hat{\omega}\right) .
$$

We define

$$
K_{j+1}=\left\{\left(q^{\prime \prime}, p^{\prime \prime},(q)_{j+1} \mid\left(x,(q)_{j}\right) \in K_{j}, q_{j+1}=q^{\prime}+R \hat{\omega}, \hat{\omega} \in G\left(x,(q)_{j}, R\right)\right\} .\right.
$$

Then, by definition of $G\left(x,(q)_{j}, R\right), K_{j+1} \subset \Gamma_{j+1}\left(t_{0}-t\right)$ and, by continuity of the free motion and the continuity of the collision $p=p^{\prime} \mapsto p^{\prime \prime}, K_{j+1}$ is compact. Since, by assumption, $r_{j+1}^{(R)}$ converges uniformly on $K_{j+1}$ to $r_{j+1}$, we conclude that

$$
\left(S^{(R)}\left(t_{2}\right) C^{(R)} S^{(R)}\left(t_{1}\right) r^{(R)}\right)_{j} \longrightarrow\left(S\left(t_{2}\right) C S\left(t_{1}\right) r\right)_{j}
$$

uniformly on $K_{j}$ as $R \rightarrow 0$.

Proof of Theorem 2. By induction to Lemma $2\left(S^{(R)}\left(t-t_{1}\right) C^{(R)} \ldots C^{(R)} S\left(t_{k}\right) r^{(R)}\right)_{J}$ con- 
verges to $\left(S\left(t-t_{1}\right) C \ldots C S\left(t_{k}\right) r\right)_{j}$ uniformly on compact sets of $\Gamma_{j}\left(t_{0}\right)$. Therefore, by the uniform bound given by Theorem 1 and by assumption $(1),\left(U_{t}^{(R)} r^{(R)}\right)_{j}$ converges to

$$
\sum_{k=0}^{\infty} \int_{0 \leqq t_{k} \leqq \ldots \leqq t_{1} \leqq t} d t_{1} \ldots d t_{k}\left(S\left(t-t_{1}\right) C \ldots C S\left(t_{k}\right) r\right)_{j}=\left(U_{t} r\right)_{j}
$$

uniformly on compact sets of $\Gamma_{j}\left(t_{0}\right)$ as $R \rightarrow 0$

\section{Convergence of the Lorentz Process}

Let $X_{j}=C\left(\Lambda_{j}\right)_{\mathrm{symm}}$ and let $X_{z}$ be the Banach space of sequences $\rho=\left(\rho_{0}, \rho_{1}, \ldots\right)$ with $\rho_{j} \in X_{j}$ and sup sup $z^{-j} \mid \rho_{j}\left((q)_{j} \mid=\|\rho\|_{z}<\infty . \rho_{j}\right.$ is symmetric in $\left(q_{1}, \ldots, q_{j}\right)$. Let $\mathscr{M}_{z}$ be the set of symmetric probability measures on $\Gamma$ such that its correlation functions (defined as in (2.3)) are in $X_{z}$. We can now state our main result.

Theorem 3. (i) Let the potential $V$ satisfy $\left({ }^{*}\right)$. Let $\mu^{(R)} \in \mathscr{M}_{z / R^{2}}, R \rightarrow 0$, be a sequence of probability measures on $\Gamma$ such that the probability that two scatterers are closer than $R$ is zero and such that its correlation functions $\rho^{(R)} \in X_{z / R^{2}}$ satisfy

(1) $\left\|\rho^{(R)}\right\|_{z / R^{2}}$ is uniformly bounded in $R$,

(2) $R^{2 j} \rho_{j}^{(R)}$ converges to $r_{j}$ with $r \in X_{z}$ uniformly on compact sets of $\Gamma_{j}=\left\{(q)_{j} \in\right.$ $\left.\Lambda^{j} \mid q_{i} \neq q_{i^{\prime}}, i, i^{\prime}=1, \ldots, j\right\}$ as $R \rightarrow 0$.

Let $P^{g(R)}$ be the regular Borel probability measure on $\Omega=\underset{t \geq 0}{X}\left(\Lambda \times \dot{\mathbb{R}}^{3}\right)_{t}$ induced by the Lorentz process with respect to $\mu^{(R)}$ with starting distribution $g d x, g \in L^{1}(\Lambda \times$ $\left.\mathbb{R}^{3}\right)$. Then

$$
P^{g(R)} \rightarrow P^{g}
$$

in the weak-*topology of regular Borel measures on $\Omega$.

(ii) The coordinate representation process $\left\{\Omega, P^{g},\left\{X_{t}, t \geqq 0\right\}\right\}$ is a Markov process (in the wide sense) if and only if

$$
r_{2}\left(q_{1}, q_{2}\right)=r_{1}\left(q_{1}\right) r_{1}\left(q_{2}\right) \text {. }
$$

In that case, let the Markov semigroup $\left\{P_{t} \mid t>0\right\}$ be defined by

$$
\int d x g(x)\left(P_{t} f\right)(x)=P^{g}\left(f\left(X_{t}\right)\right), f \in C_{\infty}\left(\Lambda \times \mathbb{R}^{3}\right) .
$$

Then $\left\{P_{t} \mid t \geqq 0\right\}$ is a strongly continuous, positivity preserving contraction semigroup on $C_{\infty}\left(\Lambda \times \mathbb{R}^{3}\right)$. The domain of its generator $D$ coincides with the domain of the generator $p \frac{\partial}{\partial q}$ of $f \mapsto f \circ T_{t}^{(0)}$ and on this domain $D$ is given by

$$
(D f)(q, p)=p \frac{\partial}{\partial q} f(q, p)+r_{1}(q) \int_{(p \cdot \hat{\omega} \geqq 0)} d \sigma(\hat{\omega}) \hat{\omega} \cdot p\left\{f\left(q, p^{\prime}\right)-f(q, p)\right\}
$$

where $\hat{\omega}$ points inward for $q \in \partial \Lambda$ and where $p^{\prime}$ is outgoing momentum to the incoming momentum $p$ colliding with the potential $V$ at $-\hat{\omega}$. (4.2) are the backwards equation of the strong Markov process $\left.\left\{\Omega,\left\{P_{x} \mid x \in \Lambda \times \mathbb{R}^{3}\right\}, X_{t}\right\}\right\}$ where $P_{x}$ is the measure starting the process at $x, P^{g}=\int d x g(x) P_{x}$. 
Proof. ad(i): We have to show [9] the weak convergence of all cylinder distributions, i.e. if $0<t_{1}<\ldots<t_{n}$ and if $f_{1}, \ldots, f_{n}$ are continuous and of compact support, then we have to show the convergence of

$$
\int d x d \mu^{(R)} g(x) f_{1}\left(T_{t_{1}}^{(\cdot)} x\right) \ldots f_{n}\left(T_{t_{n}}^{(\cdot)} x\right)
$$

as $R \rightarrow 0$. We prove the case $n=2 ; n>2$ follows then by induction. Let $f: \Lambda \times$ $\mathbb{R}^{3} \rightarrow \mathbb{C}$ be continuous and of compact support. For $\rho \in X_{z}, Y_{z}, Y_{z}^{E}, \widetilde{Y}_{z}^{E}$ we use the short hand $f \rho=\left(f \rho_{0}, f \rho_{1}, \ldots\right)$. By the group property $T_{t}^{(\cdot)}$ and by the definition of the correlation functions

$$
\begin{aligned}
& \int d x d \mu^{(R)} g(x) f_{1}\left(T_{t_{1}}^{(\cdot)} x\right) f_{2}\left(T_{t_{2}}^{(\cdot)} x\right)= \\
& =\int d x d \mu^{(R)} g(x)\left(\left(f_{1}\left(f_{2} \circ T_{t_{2}-t_{1}}^{(\cdot)}\right) \circ T_{t_{1}}^{(\cdot)}\right)(x)\right. \\
& =\int d x g(x)\left(V_{-t_{1}}^{(R)}\left(f_{1}\left(V_{-t_{2}+t_{1}}^{(R)}\left(f_{2} \rho^{(R)}\right)\right)\right)_{0}(x)\right.
\end{aligned}
$$

Let $b=\sup |V(|q|)|$. If $\rho \in Y_{z}^{E}$, then $Q^{(R)} \rho,\left(Q^{(R)}\right)^{-1} \rho \in Y_{z}^{E+b}$. Therefore $V_{t}^{(R)} \rho=$ $\left(Q^{(R)}\right)^{-1} U_{t}^{(R)} Q^{(R)} \rho \in Y_{z}^{E+2 b}$. From the Dyson expansion (2.14) it follows that for $\sigma^{(R)} \in Y_{z / R^{2}}^{E}$.

$$
\left\|U_{t}^{(R)} \sigma^{(R)}\right\|_{z / R^{2}} \leqq\left\|\sigma^{(R)}\right\|_{z / R^{2}} \exp \left[z t(2 E+2 b)^{1 / 2}\right]
$$

From (2.6) and (2.7) we conclude that

$$
\begin{aligned}
& \left\|Q^{(R)} \sigma^{(R)}-\sigma^{(R)}\right\|_{z / R^{2}} \leqq c^{\prime} R\left\|\sigma^{(R)}\right\|_{z / R^{2}} \\
& \left\|\left(Q^{(R)}\right)^{-1} \sigma^{(R)}-\sigma^{(R)}\right\|_{z / R^{2}} \leqq c^{\prime} R\left\|\sigma^{(R)}\right\|_{z / R^{2}}
\end{aligned}
$$

Therefore

$$
\left\|V_{t}^{(R)} \sigma^{(R)}-U_{t}^{(R)} \sigma^{(R)}\right\|_{z / R^{2}} \leqq \tilde{c} R\left\|\sigma^{(R)}\right\|_{z / R^{2}}
$$

where $\tilde{c}$ is a constant depending only on $E, t, b$ and $z$. Let $\chi^{(R)}=\left(\chi_{0}^{(R)}, \chi_{1}^{(R)}, \ldots\right)$ with $\chi_{j}^{(R)}\left((q)_{j}\right)=0$, whenever $\left|q_{i}-q_{i^{\prime}}\right| \leqq R, i \neq i^{\prime}=1, \ldots, j$, and $\chi_{j}^{(R)}\left((q)_{j}\right)=1$ otherwise. By our assumption on $\mu^{(R)}$ we have $\rho^{(R)}=\rho^{(R)} \chi^{(R)}=\left(\rho_{0}^{(R)}, \rho_{1}^{(R)} \chi_{1}^{(R)}, \ldots\right)$. Since $f_{2}$ is of compact support, $f_{2} \rho^{(R)} \chi^{(R)} \in Y_{z / R^{2}}^{E^{\prime}}$ for some $E^{\prime}>E$. Using the estimates (4.4) and (4.6) we find

$$
\left\|V_{-t_{1}}^{(R)}\left(f_{1}\left(V_{-t_{2}+t_{1}}^{(R)}\left(f_{2} \rho^{(R)}\right)\right)\right)-U_{-t_{1}}^{(R)}\left(f_{1}\left(U_{-t_{2}+t_{1}}^{(R)}\left(f_{2} \rho^{(R)}\right)\right)\right)\right\|_{z / R^{2}} \leqq c \cdot R\left\|\rho^{(R)}\right\|_{z / R^{2}}
$$

where $c$ depends on $f_{1}, f_{2}, t_{1}, t_{2}$ and $z$, but is independent of $R$. By (1), we conclude that (4.7) goes to zero as $R \rightarrow 0$.

$f_{2} \rho^{(R)}$ satisfies the assumptions of Theorem 2 with $t_{0}=-t_{2}+t_{1}$. Therefore, $R^{2 j}\left(U_{-t_{2}+t_{1}}^{(R)}\left(f_{2} \rho^{(R)}\right)\right)_{j}$ converges to $\left(U_{-t_{2}+t_{1}}\left(f_{2} r\right)\right)_{j}$ uniformly on compact sets of $\Gamma_{j}\left(-t_{2}+t_{1}\right)$ as $R \rightarrow 0$. $\left\|f_{1}\left(U_{-t_{2}+t_{1}}^{(R)}\left(f_{2} \rho^{(k)}\right)\right)\right\|_{z / R^{2}}$ is uniformly bounded in $R$. Therefore $f_{1}\left(U_{-t_{2}+t_{1}}^{(R)}\left(f_{2} \rho^{(R)}\right)\right)$ satisfies the assumptions of Theorem 2 with $t_{0}=-t_{2}$ which implies that $R^{2 j}\left(U_{-t_{1}}^{(R)}\left(f_{1}\left(U_{-t_{2}+t_{1}}^{(R)}\left(f_{2} \rho^{(R)}\right)\right)\right)_{j}\right.$ converges to $\left(U_{-t_{1}}\left(f_{1}\left(U_{-t_{2}+t_{1}}\right.\right.\right.$ $\left.\left.\left(f_{2} r\right)\right)\right)_{j}$ uniformly on compact sets of $\Gamma_{j}\left(-t_{2}\right)$ as $R \rightarrow 0$. In particular, using (4.7), $\left(V_{-t_{1}}^{(R)} f_{1}\left(V_{-t_{2}+t_{1}}^{(R)}\left(f_{2} \rho^{(R)}\right)\right)\right)_{0}$ converges to $\left(U_{-t_{1}}\left(f_{1}\left(U_{-t_{2}+t_{1}}\left(f_{2} r\right)\right)\right)_{0}\right.$ uniformly on compact sets of $\Gamma_{0}\left(-t_{2}\right)$. Since $\Lambda \backslash \Gamma_{0}\left(-t_{2}\right)$ is the union of a finite number 
of hypersurfaces of finite area, $\int d x d \mu^{(R)} g(x) f_{1}\left(T_{t_{1}}^{(\cdot)} x\right) f_{2}\left(T_{t_{2}}^{(\cdot)} x\right)$ converges to $\int d x g(x)$ $\left(U_{-t_{1}}\left(f_{1}\left(U_{-t_{2}+t_{1}}\left(f_{2} r\right)\right)\right)_{0}(x)\right.$ as $R \rightarrow 0$ for all $g \in L^{1}\left(\Lambda \times \mathbb{R}^{3}\right)$.

ad (ii): A straight forward computation shows [5] that if $r_{2}\left(q_{1}, q_{2}\right)=r_{1}\left(q_{1}\right) r_{1}\left(q_{2}\right)$, then $r_{j}\left((q)_{j}\right)=\prod_{i=1}^{j} r_{1}\left(q_{i}\right)$. Together with (3.2) this implies

$$
\left(V_{-t} f r\right)_{j}=\left({ }_{i=1}^{P} f\right) r_{j} \text {. }
$$

Therefore

$$
\lim _{R \rightarrow 0} \int d x d \mu^{(R)} g(x) f_{1}\left(T_{t_{1}}^{(\cdot)} x\right) f_{2}\left(T_{t_{2}}^{(\cdot)} x\right)=\int d x g(x) P_{t_{1}}\left(f_{1}\left(P_{t_{2}-t_{1}} f_{2}\right)\right)(x)
$$

and by induction for $n>2$. (4.9) is the Markov property. If $r_{2}$ does not factorize, then by (3.2) for a sufficiently smooth $f$

$$
\left.\frac{d^{2}}{d t^{2}} P_{t} f\right|_{t=0} \neq D^{2} f
$$

which shows that $\left\{P_{t} \mid t \geqq 0\right\}$ does not form a semigroup. By homogeneity in time this implies that $\left\{\Omega, P^{g},\left\{X_{t}, t \geqq 0\right\}\right\}$ is not a Markov process.

The remaining assertions follow from (3.1) and (3.2) and standard results about random flight processes (cf. [10].)

Clearly, for $t \leqq 0$ the Lorentz process converges to a stochastic process which is just the one obtained for $t \geqq 0$ reflected at the time origin.

The restriction of non-overlapping of two scatterers on the sequence $\mu^{(R)}$ of probability measures is somewhat annoying. The reason is that by putting scatterers on top of each other we may produce locally a very high momentum, which results in a poor bound for $V_{t}^{(R)}$ on $Y_{z / R^{2}}\left(\left\|V_{t}^{(R)}\right\|_{z / R^{2}}\right.$ cannot be controlled well enough). There are two ways in which the result can be improved. For hard sphere scatterers $(a=R)|p|$ is conserved and $V_{t}^{(R)}$ coincides with $U_{t}^{(R)}$. Therefore, in that case there is besides (1) and (2) no further restriction on the sequence $\mu^{(R)}$. For $V \geqq 0$, we can choose a scale of spaces ${ }_{\beta} Y_{z}$ with norm

$$
\left.{ }_{\beta}\|\rho\|_{z}=\sup _{j \geqq 0} \sup _{(q)_{j} \in A^{j}} z^{-j} \int d x \exp \left[\beta \frac{p^{2}}{2}+\sum_{i=1}^{j} V\left(q-q_{i}\right)\right)\right]\left|\rho_{j}\left(x,(q)_{j}\right)\right| .
$$

Then $V_{t}^{(R)}$ and $U_{t}^{(R)}$ are bounded from ${ }_{\beta} Y_{z}$ to ${ }_{\beta^{\prime}} Y_{z}, \beta^{\prime}>\beta$, and $\lim _{R \rightarrow 0} \| V_{t}^{(R)} \rho^{(R)}-$ $U_{t}^{(R)} \rho^{(R)} \|_{z}=0$. The analysis is then just the same as in the present case. Besides (1) and (2), there is no further restriction on the sequence $\mu^{(R)}$.

In the framework of our proof the restriction to starting distributions which are absolutely continuous with respect to the Lebesgue measure cannot be removed: If we start the process at $x$ such that $T_{t}^{(0)} x$ lies in the "bad" set $\Lambda \backslash \Gamma_{0}\left(t_{0}\right)$, then $\int d \mu^{(R)} f\left(T_{t}^{(\cdot)} x\right)$ does not converge to $\left(P_{t} f\right)(x)$. The particle may collide with the wall and then, retracing its way back to $x$, with a scatterer adjoined before at $q_{1}=q+R \hat{\omega}, q \in \Lambda \backslash \Gamma_{0}\left(t_{0}\right), \hat{\omega} \cdot p \geqq 0$. This collision process is not described by $P_{t}$. However, if we have no boundary $\left(\Lambda=\mathbb{R}^{3}\right)$, then the Lorentz process starting at $x$ converges as $R \rightarrow 0$. 
The condition for the Markov property of the limiting process has an illuminating explanation: Consider the random variables $n_{\Delta}$ on $\Gamma$ giving the number of scatterers in the measurable set $\Delta \subset \Lambda$.

If $r_{2}\left(q_{1}, q_{2}\right)=r_{1}\left(q_{1}\right) r_{1}\left(q_{2}\right)$, then

$$
\lim _{R \rightarrow 0} R^{2} \int d \mu^{(R)} n_{\Delta}=\int_{\Delta} d q_{1} r\left(q_{1}\right)
$$

and

$$
\lim _{R \rightarrow 0} R^{4}\left(\int d \mu^{(R)} n_{\Delta}^{2}-\left(\int d \mu^{(R)} n_{\Delta}\right)^{2}\right)=0
$$

i.e. $R^{2} n_{\Delta}$ converges in probability to its mean. Therefore, the Markov property is equivalent to no fluctuations in the rescaled density of scatterers in any region $\Delta \subset \Lambda$.

The limiting Markov process is well known (cf. [10] and references therein). In probabilistic terms it can be described as follows: A particle leaves the point $q$ in direction $p$, speed $v=|p|$. It travels in time $t$ a distance $v t$ suffering shocks $(=$ random jumps of $p(t))$ at random times and moving freely in between shocks. The random jumps are determined by the differential cross section of the potential $V$, i.e. by the transition $p$ to $p^{\prime}$. The speed is conserved. The probability density of suffering exactly $n$ shocks at points $q_{1}, \ldots, q_{n}$ is given by

$$
\left\{\prod_{j=1}^{n} r_{1}\left(q_{j}\right)\right\} \exp \left[-\int d s r_{1}\left(\tilde{q}\left(s \mid q, q_{1}, \ldots, q_{n}, q_{0}\right)\right)\right],
$$

where $s \mapsto \tilde{q}\left(s \mid q, q_{1}, \ldots, q_{n}, q_{0}\right)$ is the path from $q$ via $q_{1}, \ldots, q_{n}$ to $q_{0}$. Thus, $r_{1}(q)$ is the collision rate at $q \in \Lambda$.

\section{Related Results}

(a) The present method works for any dimension greater than two. The boundary conditions may be more general, e.g. of stochastic type. The latter case is of interest as a model of walls with temperature. One can treat the case with no boundary condition $\left(\Lambda=\mathbb{R}^{3}\right)$. $\mu^{(R)}$ is then a sequence of probability measures on the space of locally finite configurations of scatterers, i.e. on the space of all countable subsets of $\mathbb{R}^{3}$ such that their intersection with any bounded region is finite (cf. [8.11]). In Section 2 and 3 one has to take then suitable infinite volume limits which are however rather trivial, since the scatterers are static. The present method can also be applied to scatterers with recoil, i.e. the particle moves through a gas of freely moving, non-interacting scatterers (Rayleigh gas).

(b) Once the random flight (or transport) process is obtained, two further limiting procedures are of interest. (1) Long time limit: This problem has been studied in great generality by Papanicolaou [10]. One considers the Markov process $\left(q^{\varepsilon}(t), p^{\varepsilon}(t)\right)$ with generator of its semigroup

$$
\left(D^{\varepsilon} f\right)(q, p)=\frac{1}{\varepsilon} p \frac{\partial}{\partial q} f(q, p)+\frac{1}{\varepsilon^{2}} r_{1}(q) \int_{\{\hat{\omega} \cdot p \geqq 0\}} d \sigma(\hat{\omega}) \hat{\omega} \cdot p\left\{f\left(q, p^{\prime}\right)-f(q, p)\right\} .
$$


The collision rate is increased and at the same time the particle travels farther in a unit of time. Then $q^{\varepsilon}(t)$ converges to a diffusion process as $\varepsilon \rightarrow 0$. (2) Small angle approximation: This corresponds to either a weak potential $\lambda V(a=0)$ or, in the case of moving scatterers, to a large mass of the moving particle. The weakening of the scattering has to be compensated by an increase of the collision rate. Il'in and Khas'minskii [12] studied a special case and obtained in the limit the Ornstein-Uhlenbeck process. (Here, a more general investigation would be desirable.)

(c) There are a number of one-dimensional results. Spitzer [13] proved that if at $t=0$ the moving hard rod scatterers are Poisson distributed with i.i.d. velocities independently of the position of the scatterer, then $q^{\varepsilon}(t)=\varepsilon q\left(\frac{t}{\varepsilon^{2}}\right)$ converges to a Wiener process as $\varepsilon \rightarrow 0$. Special cases were proved before by Jespen [14] and Harris [15]. Some generalizations can be found in Szatzschneider [16] and Major/ Szasz [17]. Holley [18] considered the same model but with a heavy particle. If $M$ is the mass of the particle, he showed that $\frac{1}{\sqrt{M}} q_{M}(M t)$ converges to the Ornstein-Uhlenbeck position process as $M \rightarrow \infty$. These results have not been extended to more than one dimension. (Hennion [19] extended Holley's result to two dimensions, but only for a process differing from the one defined by the Newtonian time evolution.) We hope that a combination of (a) and (b) allows for an extension to higher dimensions.

Acknowledgements. I am grateful to E.G.D. Cohen for suggesting the problem originally. It is a pleasure to thank G. Gallavotti and J. L. Lebowitz for many helpful and very valuable discussions.

\section{References}

1. Lorentz, H. A. : Le mouvement des électrons dans les métaux. Arch. Néerl. 10, 336 (1905); in collected papers Vol. 3, p. 180

2. Bruin, C.: Phys. Rev. Letters 29, 1670 (1972)

3. Gallavotti, G. : Phys. Rev. 185, 308 (1969)

4. Gallavotti, G.: Rigorous theory of the Boltzmann equation in the Lorentz gas. Nota Interna No. 358, Istituto di Fisica, Universita di Roma (1972)

5. Lanford III, O. E. : Time evolution of large classical systems. In: Dynamical systems (ed. J. Moser). Lecture notes in physics, Vol. 38. Berlin-Heidelberg-New York: Springer 1974

6. King, F.: BBGKY hierarchy for positive potentials. Ph.D. thesis, Department of Mathematics, University of California, Berkeley (1975)

7. Ruelle, D.: Statistical mechanics. New York: Benjamin 1969

8. Gallavotti, G., Lanford, III, O. E., Lebowitz, J. L. : J. Math. Phys. 11, 2898 (1970)

9. Nelson, E. : Ann. Math. 69, 630 (1959)

10. Papanicalaou, G. C. : Bull. AMS 81, 330 (1975)

11. Lanford III, O. E. : Commun. math. Phys. 11, 257 (1969)

12. Il'in, A. M., Khas'minskii, R. Z. : Theor. Prob. Appl. 9, 421 (1964)

13. Spitzer, F. : J. Math. Mech. 18, 973 (1969)

14. Jespen, D. W. : J. Math. Phys. 6, 405 (1965)

15. Harris, T. E. : J. Appl. Prob. 2, 322 (1965) 
16. Szatzschneider, W.: A more deterministic version of Harris-Spitzer's "random constant velocity" model for infinite systems of particles. Probability Winter School, Karpacz. Lecture notes in mathematics, Vol. 472. Berlin-Heidelberg-New York: Springer 1975

17. Major P., Szasz, D. : On the effect of collisons on the motion of an atom in $\mathbb{R}^{1}$. Preprint (1977)

18. Holley, R. : Trans. AMS 144, 523 (1969)

19. Hennion, G. : Z. Wahrscheinlichkeitstheorie verw. Gebiete 25, 123 (1973)

Communicated by J. L. Lebowitz

Received October 17, 1977

\section{Note Added in Proof}

Prof. Cohen pointed out to me that $\mathrm{H}$. van Beijeren (unpublished notes) had obtained results similar to those of Theorem 1. 